\title{
A MOS-based dynamic memetic differential evolution algorithm for continuous optimization: a scalability test
}

\author{
Antonio LaTorre · Santiago Muelas • \\ José-María Peña
}

\begin{abstract}
Continuous optimization is one of the areas with more activity in the field of heuristic optimization. Many algorithms have been proposed and compared on several benchmarks of functions, with different performance depending on the problems. For this reason, the combination of different search strategies seems desirable to obtain the best performance of each of these approaches. This contribution explores the use of a hybrid memetic algorithm based on the multiple offspring framework. The proposed algorithm combines the explorative/exploitative strength of two heuristic search methods that separately obtain very competitive results. This algorithm has been tested with the benchmark problems and conditions defined for the special issue of the Soft Computing Journal on Scalability of Evolutionary Algorithms and other Metaheuristics for Large Scale Continuous Optimization Problems. The proposed algorithm obtained the best results compared with both its composing algorithms and a set of reference algorithms that were proposed for the special issue.
\end{abstract}

Keywords Continuous optimization - Multiple offspring sampling - Scalability

\footnotetext{
A. LaTorre $(\triangle) \cdot S$. Muelas - J.-M. Peña

Department of Computer Systems Architecture and Technology,

Facultad de Informática, Universidad Politécnica de Madrid,

Madrid, Spain

e-mail: atorre@fi.upm.es

S. Muelas

e-mail: smuelas@fi.upm.es

J.-M. Peña

e-mail: jmpena@fi.upm.es
}

\section{Introduction}

Continuous optimization is a field of research which is getting more and more attention in the past years. Many real-world problems from very different domains (biology, engineering, data mining, etc.) can be formulated as the optimization of a continuous function. These problems have been tackled using evolutionary algorithms (EA) (Muelas et al. 2009) or similar metaheuristics (Tseng and Chen 2008).

Selecting an appropriate algorithm to solve a continuous optimization problem is not a trivial task. Although a particular algorithm can be configured to perform properly in a given scale of problems (considering the number of variables as their dimensionality), the behavior of the algorithm can degrade as this dimensionality increases, even if the nature of the problem remains the same.

In this contribution, the multiple offspring sampling (MOS) framework has been used to combine a differential evolution (DE) algorithm and the first one of the local searches of the MTS algorithm (through the rest of this paper we will refer to this local search as MTS-LS1, and its pseudocode as well as an explanation of how it works can be found in Tseng and Chen (2008)). This framework allows the combination of different metaheuristics following a high-level relay hyrbid (HRH) approach (this nomenclature will be reviewed in Sect. 2) in which the number of evaluations that each algorithm can carry out is dynamically adjusted. It will be shown that a MOS-based algorithm actually obtains the best results compared with its composing algorithms and a set of reference algorithms that were proposed for the special issue: DE, Real-Coded CHC and G-CMA-ES, the best algorithm of the "Special Session on Real-Parameter Optimization" held at the CEC 2005 Congress. The set of problems that were used for the 
tests is based on the benchmark of the "Special Session and Competition on Large Scale Global Optimization" held at the CEC 2008 congress (Tang et al. 2007), and a set of additional problem functions of different difficulty types. The experiments were carried out on a wide range of dimensions in order to evaluate the behavior of the algorithms as the number of variables increases.

The rest of the paper is organized as follows: In Sect. 2, relevant related work is briefly reviewed. Section 3 details the proposed algorithm. In Sect. 4 the experimental scenario is described. Section 5 presents and comments on the results obtained and lists the most relevant facts from this analysis. Finally, Sect. 6 contains the concluding remarks obtained from this work.

\section{Related work}

The HRH terminology was introduced in Talbi (2002), one of the first attempts to define a complete taxonomy of hybrid metaheuristics. This taxonomy is a combination of a hierarchical and a flat classification structured into two levels. The first level defines a hierarchical classification in order to reduce the total number of classes, whereas the second level proposes a flat classification, in which the classes that define an algorithm may be chosen in an arbitrary order. From this taxonomy, the following four basic hybridization strategies can be derived: (a) LRH (low-level relay hybrid): one metaheuristic is embedded into a single-solution metaheuristic. (b) HRH (high-level relay hybrid): two metaheuristics are executed in sequence. (c) LTH (low-level teamwork hybrid): one metaheuristic is embedded into a population-based metaheuristic. (d) HTH (high-level teamwork hybrid): two metaheuristics are executed in parallel.

For this work, we have focused on the HRH group, the one the algorithm proposed in this paper belongs to.

In the past years there has been an intense research in HRH or memetic models, combining different types of metaheuristics. In particular, the DE algorithm is one of the EA that has been recently hybridized using this kind of strategy. In the following paragraphs some of the most recent and representative approaches will be reviewed.

Gao and Wang (2007) proposed CSDE1, a memetic DE, to optimize thirteen 30-dimensional continuous problems. CSDE1 uses simplex (Nelder-Mead method) to carry out the local search (LS) using also chaotic systems to create the initial population. CSDE1 applies the local search only to the best individual in the population at each generation.

Tirronen et al. (2007) designed a hybrid DE algorithm that combined the Hooke-Jeeves Algorithm (HJA) and the stochastic local search (SLS), coordinated by an adaptive rule that estimates fitness diversity using the ratio between the standard deviation and the average fitness of the population. This algorithm was compared against a regular DE and an evolution strategy (ES) on the problem of weighting coefficients to detect defects in paper production.

The fast adaptive memetic algorithm (FAMA) (Caponio et al. 2007), proposed by Caponio et al., is a memetic algorithm with a dynamic parameter setting and two local searchers adaptively launched, either one by one or simultaneously, according to the needs of the evolution. The employed local search methods are the Hooke-Jeeves and the Nelder-Mead methods. The Hooke-Jeeves method is executed only on the elite individual, whereas the Nelder-Mead simplex is carried out on 11 randomly selected individuals. FAMA includes a self-adaptive criterium based on a fitness diversity measure and the iteration number. Mutation probability and other search parameters depend also on the diversity measure. the FAMA algorithm was compared against Tirronen's algorithm and SFMDE obtaining better results for the problem of permanent magnet synchronous motors (Caponio et al. 2008).

There have also been some studies that have tried to use adaptive learning to combine the algorithms. Ong and Keane (2004), propose two adaptive strategies, one heuristic and one stochastic, to adapt the participation of several local searches when combined with a genetic algorithm (GA). In both strategies, there is a learning phase in which the performance of each local search is stored and used in later generations in order to select the local search to apply. Several local searches are combined with a metaheuristic algorithm using also an adaptive scheme in Caponio et al. (2009) and Tirronen et al. (2008). The application of each algorithm is based on a population diversity measure which varies among the studies. When applied to the DE algorithm, this strategy prevents the stagnation problems of the DE by reducing the excessive difference between the best individual and the rest of the population. A quite different approach is followed by LaTorre et al. (2010). In this work, a hybrid algorithm combines several genetic algorithms in such a way that their contribution to the overall search is learned through the use of Reinforcement Learning techniques. Moreover, the hybridization strategy learned in one execution of the algorithm is used and refined in further executions.

In the recent years, there have been several sessions that have focused on continuous optimization. At the CEC 2005 Congress, a "Special Session on Real-Parameter Optimization" was carried out for analyzing the performance of several algorithms on a benchmark of 25 continuous functions (Suganthan et al. 2005). In this competition, the G-CMA-ES algorithm obtained the best results among all the evaluated techniques. However, the use of a covariance matrix makes it not appropriate for high-dimensional functions. Differential evolution showed to be a competitive 
alternative in ten dimensions: $\mathrm{SaDE}$, a self-adaptive $\mathrm{DE}$ (Qin and Suganthan 2005), obtained the third position in the final function value, and a real-coded $\mathrm{DE}$ algorithm (Rönkkönen et al. 2005) was ranked third in success performance.

On the other hand, the "Special Session and Competition on Large Scale Global Optimization", held at the CEC 2008 Congress, focused on seven functions solved in 100 , 500, and 1,000 dimensions (Tang et al. 2007). The MTS algorithm (Tseng and Chen 2008), which combines several local searches using a small population, was the best algorithm of the competition.

Considering these two results, an intuitive approach would be to combine a local search algorithm, in particular the first one of those proposed by MTS, with a DE algorithm. This hybrid approach could be seen as the equivalent to the memetic algorithms in classical genetic algorithms.

For this reason, an HRH algorithm combining these two algorithms was presented by Muelas et al. (2009) at the Workshop on Evolutionary Algorithms and other Metaheuristics for Continuous Optimization Problems-A Scalability Test held at the ISDA 2009 Conference. In this algorithm, the hybridization with an exploitative local search helps the DE to avoid the stagnation problem. On the other hand, the DE allows the local search to find promising regions with a moderate consumption of fitness evaluations. This algorithm proved to deal successfully with problems of different dimensionality and obtained one of the best results of the workshop.

\section{Proposal}

In this section, the MOS framework is reviewed (Sect. 3.1) and the proposed algorithm is presented (Sect. 3.2).

\subsection{Multiple offspring sampling in brief}

One of the main advantages of metaheuristics, in general, and EAs, in particular, is their flexibility and adaptability, which allows its application to a wide range of optimization problems in different domains. However, this characteristic that makes EAs suitable for solving even the hardest optimization problems with a remarkable success, is, paradoxically, one of the most important matters that somebody interested in using these algorithms for his research has to deal with. An appropriate selection of a single algorithm and its associated parameters for a particular optimization problem is a difficult issue (Grefenstette (1986), states that this task sometimes becomes an optimization problem itself).

The No Free Lunch Theorem (Wolpert and Macready 1997) holds that "any two algorithms are equivalent when their performance is averaged across all possible problems". In other words, this means that it is impossible to define a general strategy that outperforms any other algorithm for every possible problem. This, of course, applies for EA. Even if an EA has been proved to be successful on a similar problem, this does not guarantee that this success will be repeated. A slight variation on the conditions or the data used in the experimentation could lead to unpredictable results, much of the times not as satisfactory as expected.

Additionally, several authors report that the hybridization of different EAs, encodings or operators can significantly boost the performance of the hybrid approach (Caruana and Schaffer 1988; Mladenovic and Hansen 1997; Schnier and Yao 2000; Thierens 2005; Whitacre et al. 2006; Lin et al. 2007). This opens new alternatives to improve the performance by the combination of different evolutionary approaches and means that now, not only every single alternative should be considered, but also all the combinations of any of them.

For this reason, we review in this work the MOS framework for the development of Dynamic Hybrid EAs (LaTorre et al. 2009). MOS provides the functional formalization necessary to design the aforementioned algorithms, as well as the tools to identify and select the best performing configuration for the problem under study. In this context, the hybridization of several algorithms can lead to the following two situations:

- A collaborative synergy emerges among the different algorithms that improves the performance of the best one when it is used individually.

- A competitive selection of the best one takes place, in which a similar performance (often the same) is obtained with a minimum overhead.

The hybrid algorithms developed with MOS share many of the characteristics of traditional EAs. However, there are some differences between both groups of algorithms. In MOS, a key term is the concept of technique, which is a mechanism, decoupled from the main algorithm, to generate new candidate solutions. This means that, within a MOS-based algorithm, several offspring mechanisms can be used simultaneously, and it is the main algorithm which selects among the available optimization techniques the most appropriate for the particular problem and search phase. A more concrete definition for these offspring mechanisms follows:

Definition 1 In MOS, an offspring technique is a mechanism to create new individuals in which(a) a particular evolutionary algorithm model, (b) an appropriate solution encoding, (c) specific operators (if required), and (d) necessary parameters have been defined. 
Furthermore, the use of multiple offspring mechanisms simultaneously has to be controlled in some way. The MOS framework offers two groups of functions to deal with this issue: quality and participation functions. The first group of functions evaluate how good a set of new individuals is from the point of view of a desirable characteristic. The second group of functions consider a series of quality values computed by the first group and adjust the number of new individuals that each offspring technique will be allowed to generate in the next step of the search. This way, the algorithm is able to dynamically adjust the participation of each of the available techniques and exploit the benefits of each of them at different stages of the search process.

Finally, the MOS framework allows the development of both HTH and HRH algorithms (according to Talbi's nomenclature seen before).

In the case of the HTH algorithms, the main algorithm manages an overall population which is shared by all the techniques. At each generation, the participation of each of the available techniques is adjusted according to the quality of the solutions created in the previous generation. Each of these techniques will produce a percentage of the overall shared population. A pseudocode of this approach is given in Algorithm 1. In this pseudocode, $P_{i}$ represents the overall shared population at generation $i, T^{(j)}$ is technique $j, O_{i}^{(j)}$ the offspring subpopulation produced by technique $j$ at generation $i, Q_{i}^{(j)}$ the quality value associated to each $O_{i}^{(j)}$ subpopulation, $\Pi_{i}^{(j)}$ the participation ratio (percentage of individuals of the overall shared population) that technique $j$ can produce at generation $i$ and $P F$ and $Q$ the participation and quality functions, respectively. Original and offspring populations are combined by using any of the existing elitism procedures, from classic to full elitism, which most of the times offers the best performance in terms of quality of the solutions.

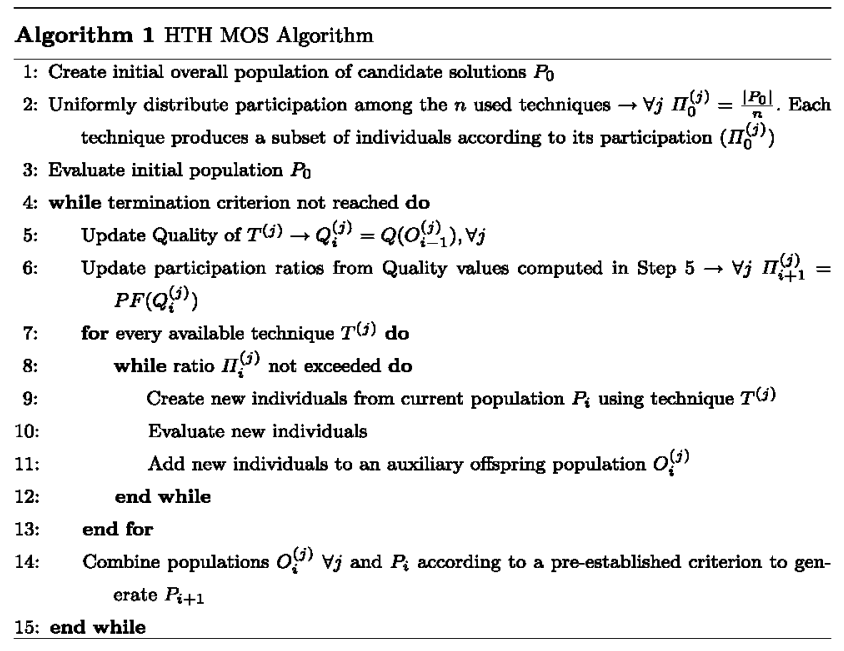

On the other hand, in the case of the HRH algorithms the available techniques are used in sequence, one after the other, each of them reusing the output population of the previous technique. This approach fits better when there are non-population-based techniques, such as local searches, as these techniques are not constrained to produce a percentage of the common population. In this case, the search process is divided into a fixed number of steps that is established at the beginning of the execution. Each step is assigned a constant amount of fitness evaluations ( $F E s_{i}$ in Algorithm 2), which are distributed by the participation function (PF). Each technique can manage its number of allocated FEs at each step of the algorithm $\left(F E s_{i}^{(j)}\right)$ in its own particular way. For example, a population-based technique, such as DE, could execute several iterations of the algorithm, whereas a local search could decide to spend all its assigned evaluations in improving just one individual. The quality of the new individuals of each technique will be averaged at the end of the whole set of evaluations of that step, as the division of the search into generations depends on each of the techniques. A pseudocode of this approach is given in Algorithm 2.

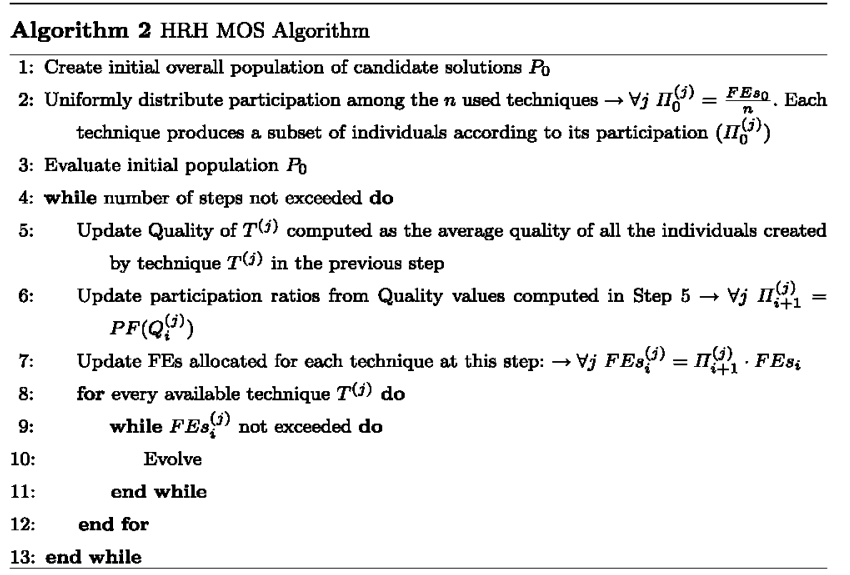

\subsection{Proposed algorithm}

In this contribution, an HRH dynamic memetic DE algorithm is proposed. This algorithm combines the explorative/exploitative strength of two heuristic search methods that separately obtain very competitive results in either low- or high-dimensional problems. An HRH approach has been preferred to an HTH one as it is more natural to allow each technique to use its assigned FEs in its own way instead of forcing all the techniques to use a populationbased scheme. Additionally, both algorithms work better when they can execute for a longer time. Making them compete for a percent of the offspring population of each 
generation would lead to a situation in which each technique would hinder the normal behavior of the other one, resulting in a poor performance of the hybrid algorithm.

For the adjustment of the participation of each technique in the overall search process, a new quality function $(\mathrm{QF})$ has been proposed. This QF takes into account two desirable characteristics in a search algorithm: the average fitness increment of the newly created individuals after a set of allocated fitness evaluations and the number of times that these improvements take place (Eq. 1).

$Q_{i}^{(j)}=\left\{\begin{array}{lll}\Sigma_{i-1}^{(j)} & \text { if } \forall k, l \in[1, n]: & \Sigma_{i-1}^{(k)}>\Sigma_{i-1}^{(l)} \\ & & \Gamma_{i-1}^{(k)}>\Gamma_{i-1}^{(l)} \\ \Gamma_{i-1}^{(j)} & \text { otherwise } & \end{array}\right.$

$Q_{i}^{(j)} \equiv$ Quality of technique $T^{(j)}$ in step $i$

$\Sigma_{i}^{(j)} \equiv$ Average fitness increment of $T^{(j)}$ in step $i$ $\Gamma_{i}^{(j)} \equiv$ Number of fit. Improvements of $T^{(j)}$ in step $i$

This quality function uses the average fitness increment as the effective QF only if there is consensus between both measures. If this is not the case, the raw number of fitness improvements is used. The logic behind this function is that, in some functions, the use of the average fitness increment QF could be very elitist. In some particular situations, a technique which is not carrying out an effective search could introduce, for some reason, a large increment in the average fitness value of the new individuals. This could be due, for example, to a recombination of poor solutions. In such a case, it is easy for a technique to improve previous solutions. However, it could be more adequate to carry out small changes to good individuals in order to find the right "path" to the global optimum rather than carrying out substantial modifications to poor solutions. For this reason, a consensus of both measures is required in order to apply the more elitist average fitness increment QF. If this is not the case, the number of fitness improvements is used to guarantee a softer adjustment of participation.

The quality values computed by this QF are used by a dynamic participation function to adjust the number of fitness evaluations allocated for each technique at each step (Eq. 2). This PF computes, at each step, a trade-off factor for each technique, $\Delta_{i}^{(j)}$, that represents the decrease in participation for the $j$-th technique at the $j$-th step, for every technique except the best performing ones. These techniques will increase their participation by the sum of all those $\Delta_{i}^{(j)}$ divided by the number of techniques with the best quality values.

$$
\begin{aligned}
P F_{\text {dyn }}\left(Q_{i}^{(j)}\right) & =\left\{\begin{array}{cc}
\Pi_{i-1}^{(j)}+\eta & \text { if } j \in \text { best } \\
\Pi_{i-1}^{(j)}-\Delta_{i}^{(j)} & \text { otherwise }
\end{array}\right. \\
\eta & =\frac{\sum_{k \notin \text { best }} \Delta_{i}^{(k)}}{\mid \text { best } \mid} \\
\text { best } & =\left\{l / Q_{i}^{(l)} \geq Q_{i}^{(m)} \forall l, m \in[1, n]\right\}
\end{aligned}
$$

The aforementioned $\Delta_{i}^{(j)}$ values are computed as shown in Eq. 3. These $\Delta_{i}^{(j)}$ factors are computed from the relative difference between the quality of the best and the $j$-th techniques, $n$ being the number of available techniques. In this equation, $\xi$ represents a reduction factor, i.e., the ratio that is transferred from one technique to the other(s) $(0.05$ in this experimentation). Finally, a minimum participation ratio can be established to guarantee that all the techniques are represented through all the search. This is done to avoid, if possible, premature convergence to undesired solutions caused by a technique that obtains all the participation in the early steps of the search and quickly converges to poor regions of the solution space, preventing the other techniques to collaborate at later stages of the process, in which they could be more beneficial.

$$
\Delta_{i}^{(j)}=\xi \cdot \frac{Q_{i}^{(\text {best })}-Q_{i}^{(j)}}{Q_{i}^{(\text {best })}} \cdot \Pi_{i-1}^{(j)} \quad \forall j \in[1, n] / j \neq \text { best }
$$

Additionally, a population reset method has been also included in order to avoid a particular situation that has been observed in some functions in which the whole population converges to a local optimum very close to the global optimum. In those cases, all the solutions but one (the best one, as all the solutions are the same) are uniformly re-initialized. This way, the algorithm can reach the global optimum more easily.

To summarize, the HRH Memetic DE works as follows: All the available techniques are allocated the same number of FEs at the beginning of the execution. At the end of each step, the quality of the new solutions created by each technique is evaluated and, based on this quality, its participation ratio is adjusted accordingly. This participation ratio is used to compute the number of FEs that each technique will be allowed to use in the next step of the search. If a minimum participation ratio has been established, then the number of FEs can not go below this threshold. If the whole population converges to the same solution, it is reset preserving one copy on the best solution found so far, to allow the algorithm to converge to the global optimum. 
Table 1 Computer configuration

\begin{tabular}{ll}
\hline $\mathrm{PC}$ & Intel Xeon 8 cores $1.86 \mathrm{Ghz} \mathrm{CPU}$ \\
Operating system & Ubuntu Linux 8.04 \\
Prog. language & $\mathrm{C}++$ \\
Compiler & $\mathrm{GNU} \mathrm{C}++4.3 .2$
\end{tabular}

\section{Experimentation}

\subsection{Benchmark suite}

A total of 19 continuous optimization functions have been considered for this experimentation. The first six functions were originally proposed for the "Special Session and Competition on Large Scale Global Optimization" held at the CEC 2008 Congress (Tang et al. 2007). The next five functions were proposed for the Workshop on Evolutionary Algorithms and other Metaheuristics for Continuous Optimization Problems-A Scalability Test held at the ISDA 2009 Conference. Finally, the last seven functions are non-separable functions built by combining two functions belonging to the set of functions $f 1-f 11$. All the functions are completely scalable functions, which makes possible the scalability test proposed for this special issue. A detailed description of the selected benchmark can be found at the web page of the organizers of the special issue. ${ }^{1}$

The results reported for this work are the average of 25 independent executions conducted on the computer configuration displayed in Table 1. For each function, five different numbers of dimensions have been tested: $D=$ $50, D=100, D=200, D=500$ and $D=1,000$ with a maximum number of fitness evaluations fixed to $5,000^{*}$ dimension. In order to allow an easy comparison with the composing algorithms of the hybrid approach presented in this work, as well as with the MDE-DC algorithm presented in Muelas et al. (2009), the average errors for these three algorithms are also provided. As suggested by the organizers of this special issue, all the error values below $1 \mathrm{e}-14$ have been rounded to zero for all the algorithms.

\subsection{Parameter tuning}

The parameters of the DE technique were selected according to the extensive parameter tuning that was carried out for the HRH algorithm presented in Muelas et al. (2009). Regarding the MTS-LS1 technique, it was configured as suggested in the original paper (see (Tseng and Chen 2008) for further details). Both algorithms, when used independently, use the same configuration. For this study, both the population size and the number of steps

\footnotetext{
${ }^{1}$ http://sci2s.ugr.es/eamhco/CFP.php.
}

Table 2 Configuration of the MOS-based algorithm

\begin{tabular}{ll}
\hline Parameter & Value \\
\hline Population size & 15 \\
DE CR & 0.5 \\
DE F & 0.5 \\
DE crossover operator & Exponential \\
DE selection operator & Tournament 2 \\
DE model & Classic \\
Minimum participation ratio & $5 \%$ \\
Number of steps & 84 \\
\hline
\end{tabular}

parameters were tuned in order to improve the performance of the algorithm. The population size values that were explored vary from 10 up to 40 individuals with increments of 5 individuals. For the number of steps parameter, the limits were from 50 up to 101 steps with increments of 17 steps, which are roughly equivalent to a $2 \%, 1.5 \%, 1.2 \%$ and $1 \%$ of the overall number of available FEs, respectively. Table 2 displays the final values that were selected for the algorithm.

Finally, in this work the Differential Evolution technique has been always chosen as the first algorithm to execute at the beginning of each step. It seemed reasonable to execute first the DE in order to provide the LS with a set of already good solutions instead of carrying out the LS on a random set of solutions (this could be especially important at the beginning of the execution of the algorithm). However, none other policy has been considered: using always the LS at the beginning of each step, alternating both algorithms, choosing randomly, etc. In a further research, several policies will be tested in order to check if the order in which the algorithms are executed is relevant for the performance of the hybrid algorithm.

\section{Analysis of the results}

In this section, the experimental results obtained with the proposed algorithm are thoroughly analyzed and validated. In particular, a statistical analysis on the average error is conducted, comparing the proposed algorithm against (i) its composing algorithms, (ii) the MDE-DC algorithm, seed of this work, presented in Muelas et al. (2009), and (iii) the reference algorithms specified for this special issue. To continue, the scalability behavior of the proposed algorithm is analyzed, from the point of view of the evolution of the achieved accuracy as the complexity (i.e. the number of dimensions) of the function grows. Furthermore, the computational running time of the MOS-based hybrid algorithm is reported and discussed. Finally, an analysis on how the participation of each algorithm is adjusted and 
which quality function is being used at each moment is also provided.

\subsection{Statistical analysis}

Tables 3, 4, 5, 6, 7 contain the average error, for each function and dimension, of the MOS-based hybrid algorithm, both composing algorithms used separately (DE and MTS-LS1) and the MDE-DC hybrid algorithm. The individual DE and MTS-LS1 were run with the same configuration of the MOS algorithm, presented in Table 2 (for the parameters that apply in each case), and the MDE-DC algorithm was executed with the best configuration found in its own parameter tuning process (see Muelas et al. 2009 for details). At the bottom of each table, the total number of functions solved by each algorithm to the aforementioned precision is reported. As can be seen, the new Dynamic MOS-based algorithm solves the highest number of functions at any dimension. The difference with regards the other three algorithms increases as the number of dimensions grows.

It is important to highlight the fact that, in 100 dimensions, the average error for $f 14$ is not zero, as it is in all the other dimensions. This is due to the convergence problem to a local optimum commented in Sect.,3.2, that still happens, although with a smaller probability, even if the population reset mechanism is being used. However, it is important to state that only 1 of the 25 independent executions present this problem. The remaining executions converge to the global optimum with a precision higher than $1 \mathrm{e}-20$.

Once the average errors have been presented, a statistical analysis following the guidelines proposed in García et al. (2009) can be carried out. This analysis will be conducted on two groups of algorithms. First, the proposed algorithm is compared with its composing techniques and the MDE-DC algorithm, to prove that this dynamic hybridization approach is better than the sole use of DE and MTS-LS1 and also than a static combination of these two algorithms (MDE-DC). And second, the MOS-based algorithm is compared with the reference algorithms: G-CMA-ES, CHC and two DE algorithms with exponential and binomial crossover, respectively. This comparison has been done in two steps, considering the average errors up to 500 and 1,000 dimensions, respectively. This is due to the lack of results for the G-CMA-ES algorithm in 1,000 dimensions as a consequence of the extremely large computation time required to complete those executions.

As for the first statistical comparison, Table 8 shows the average ranking of MOS, MDE-DC, MTS-LS1, and DE on the whole set of functions and dimensions. We can see that the MOS algorithm obtains the best average ranking, followed by the other hybrid algorithm, MDE-DC. The two individual algorithms obtained the worst results.
Table 3 Average error on 50-D functions

\begin{tabular}{lllll}
\hline Function & MOS & MDE-DC & DE & MTS-LS1 \\
\hline Sphere & $0.00 \mathrm{e}+00$ & $0.00 \mathrm{e}+00$ & $1.50 \mathrm{e}+00$ & $0.00 \mathrm{e}+00$ \\
Schwefel 2.21 & $4.64 \mathrm{e}-13$ & $8.89 \mathrm{e}-11$ & $4.16 \mathrm{e}+01$ & $8.84 \mathrm{e}-11$ \\
Rosenbrock & $9.61 \mathrm{e}+00$ & $1.24 \mathrm{e}+01$ & $5.31 \mathrm{e}+01$ & $1.63 \mathrm{e}+02$ \\
Rastrigin & $0.00 \mathrm{e}+00$ & $2.38 \mathrm{e}-01$ & $1.58 \mathrm{e}+00$ & $0.00 \mathrm{e}+00$ \\
Griewank & $0.00 \mathrm{e}+00$ & $0.00 \mathrm{e}+00$ & $9.52 \mathrm{e}-02$ & $7.68 \mathrm{e}-03$ \\
Ackley & $0.00 \mathrm{e}+00$ & $0.00 \mathrm{e}+00$ & $4.18 \mathrm{e}-01$ & $0.00 \mathrm{e}+00$ \\
Schwefel 2.22 & $0.00 \mathrm{e}+00$ & $0.00 \mathrm{e}+00$ & $0.00 \mathrm{e}+00$ & $0.00 \mathrm{e}+00$ \\
Schwefel 1.2 & $1.54 \mathrm{e}-08$ & $3.67 \mathrm{e}-01$ & $1.98 \mathrm{e}+01$ & $9.56 \mathrm{e}-12$ \\
Extended $f 10$ & $0.00 \mathrm{e}+00$ & $0.00 \mathrm{e}+00$ & $2.07 \mathrm{e}-03$ & $1.03 \mathrm{e}+02$ \\
Bohachevsky & $0.00 \mathrm{e}+00$ & $0.00 \mathrm{e}+00$ & $0.00 \mathrm{e}+00$ & $0.00 \mathrm{e}+00$ \\
Schaffer & $0.00 \mathrm{e}+00$ & $0.00 \mathrm{e}+00$ & $0.00 \mathrm{e}+00$ & $1.04 \mathrm{e}+02$ \\
$f 12$ & $0.00 \mathrm{e}+00$ & $0.00 \mathrm{e}+00$ & $4.08 \mathrm{e}+00$ & $1.34 \mathrm{e}+01$ \\
$f 13$ & $4.55 \mathrm{e}-01$ & $6.71 \mathrm{e}-01$ & $2.82 \mathrm{e}+01$ & $2.94 \mathrm{e}+01$ \\
$f 14$ & $0.00 \mathrm{e}+00$ & $1.98 \mathrm{e}-01$ & $1.24 \mathrm{e}+00$ & $5.52 \mathrm{e}+01$ \\
$f 15$ & $0.00 \mathrm{e}+00$ & $0.00 \mathrm{e}+00$ & $0.00 \mathrm{e}+00$ & $0.00 \mathrm{e}+00$ \\
$f 16$ & $0.00 \mathrm{e}+00$ & $0.00 \mathrm{e}+00$ & $4.38 \mathrm{e}-01$ & $4.06 \mathrm{e}+01$ \\
$f 17$ & $1.40 \mathrm{e}+01$ & $6.12 \mathrm{e}+00$ & $7.59 \mathrm{e}+00$ & $2.17 \mathrm{e}+02$ \\
$f 18$ & $0.00 \mathrm{e}+00$ & $1.19 \mathrm{e}-01$ & $4.48 \mathrm{e}-01$ & $5.65 \mathrm{e}+01$ \\
$f 19$ & $0.00 \mathrm{e}+00$ & $0.00 \mathrm{e}+00$ & $8.39 \mathrm{e}-02$ & $0.00 \mathrm{e}+00$ \\
Solved funcs. & 14 & 11 & 4 & 7 \\
\hline & & & & \\
\hline
\end{tabular}

Table 4 Average error on 100-D functions

\begin{tabular}{lllll}
\hline Function & MOS & MDE-DC & DE & MTS-LS1 \\
\hline Sphere & $0.00 \mathrm{e}+00$ & $0.00 \mathrm{e}+00$ & $3.79 \mathrm{e}+00$ & $1.09 \mathrm{e}-12$ \\
Schwefel 2.21 & $2.94 \mathrm{e} 12$ & $7.13 \mathrm{e}-09$ & $7.58 \mathrm{e}+01$ & $4.66 \mathrm{e}-10$ \\
Rosenbrock & $2.03 \mathrm{e}+01$ & $1.38 \mathrm{e}+01$ & $1.27 \mathrm{e}+02$ & $2.32 \mathrm{e}+02$ \\
Rastrigin & $0.00 \mathrm{e}+00$ & $1.19 \mathrm{e}-01$ & $2.85 \mathrm{e}+00$ & $1.05 \mathrm{e}-12$ \\
Griewank & $0.00 \mathrm{e}+00$ & $0.00 \mathrm{e}+00$ & $3.05 \mathrm{e}-01$ & $6.70 \mathrm{e}-03$ \\
Ackley & $0.00 \mathrm{e}+00$ & $0.00 \mathrm{e}+00$ & $4.34 \mathrm{e}-01$ & $1.20 \mathrm{e}-12$ \\
Schwefel 2.22 & $0.00 \mathrm{e}+00$ & $0.00 \mathrm{e}+00$ & $0.00 \mathrm{e}+00$ & $0.00 \mathrm{e}+00$ \\
Schwefel 1.2 & $9.17 \mathrm{e}-02$ & $1.13 \mathrm{e}+01$ & $4.74 \mathrm{e}+02$ & $1.43 \mathrm{e}-03$ \\
Extended $f 10$ & $0.00 \mathrm{e}+00$ & $0.00 \mathrm{e}+00$ & $3.71 \mathrm{e}-03$ & $2.20 \mathrm{e}+02$ \\
Bohachevsky & $0.00 \mathrm{e}+00$ & $0.00 \mathrm{e}+00$ & $0.00 \mathrm{e}+00$ & $0.00 \mathrm{e}+00$ \\
Schaffer & $0.00 \mathrm{e}+00$ & $0.00 \mathrm{e}+00$ & $8.58 \mathrm{e}-04$ & $2.10 \mathrm{e}+02$ \\
$f 12$ & $0.00 \mathrm{e}+00$ & $0.00 \mathrm{e}+00$ & $2.71 \mathrm{e}+00$ & $3.91 \mathrm{e}+01$ \\
$f 13$ & $1.75 \mathrm{e}+01$ & $2.16 \mathrm{e}+00$ & $5.87 \mathrm{e}+01$ & $1.75 \mathrm{e}+02$ \\
$f 14$ & $1.68 \mathrm{e}-11$ & $4.46 \mathrm{e}-01$ & $2.21 \mathrm{e}+00$ & $2.04 \mathrm{e}+02$ \\
$f 15$ & $0.00 \mathrm{e}+00$ & $0.00 \mathrm{e}+00$ & $0.00 \mathrm{e}+00$ & $0.00 \mathrm{e}+00$ \\
$f 16$ & $0.00 \mathrm{e}+00$ & $0.00 \mathrm{e}+00$ & $3.52 \mathrm{e}+00$ & $1.04 \mathrm{e}+02$ \\
$f 17$ & $1.43 \mathrm{e}+01$ & $8.51 \mathrm{e}+00$ & $1.58 \mathrm{e}+01$ & $4.17 \mathrm{e}+02$ \\
$f 18$ & $0.00 \mathrm{e}+00$ & $3.97 \mathrm{e}-01$ & $8.76 \mathrm{e}-01$ & $1.22 \mathrm{e}+02$ \\
$f 19$ & $0.00 \mathrm{e}+00$ & $0.00 \mathrm{e}+00$ & $0.00 \mathrm{e}+00$ & $0.00 \mathrm{e}+00$ \\
Solved funcs. & 13 & 11 & 4 & 4 \\
\hline
\end{tabular}


Table 5 Average error on 200-D functions

\begin{tabular}{lllll}
\hline Function & MOS & MDE-DC & DE & MTS-LS1 \\
\hline Sphere & $0.00 \mathrm{e}+00$ & $0.00 \mathrm{e}+00$ & $8.55 \mathrm{e}+00$ & $2.29 \mathrm{e}-12$ \\
Schwefel 2.21 & $1.24 \mathrm{e}-11$ & $3.75 \mathrm{e}-09$ & $1.05 \mathrm{e}+02$ & $4.54 \mathrm{e}-09$ \\
Rosenbrock & $4.01 \mathrm{e}+01$ & $2.29 \mathrm{e}+01$ & $3.32 \mathrm{e}+05$ & $1.69 \mathrm{e}+02$ \\
Rastrigin & $0.00 \mathrm{e}+00$ & $1.19 \mathrm{e}-01$ & $6.98 \mathrm{e}+00$ & $2.34 \mathrm{e}-12$ \\
Griewank & $0.00 \mathrm{e}+00$ & $0.00 \mathrm{e}+00$ & $4.05 \mathrm{e}-01$ & $5.42 \mathrm{e}-03$ \\
Ackley & $0.00 \mathrm{e}+00$ & $0.00 \mathrm{e}+00$ & $7.14 \mathrm{e}-01$ & $2.38 \mathrm{e}-12$ \\
Schwefel 2.22 & $0.00 \mathrm{e}+00$ & $0.00 \mathrm{e}+00$ & $0.00 \mathrm{e}+00$ & $0.00 \mathrm{e}+00$ \\
Schwefel 1.2 & $1.16 \mathrm{e}+02$ & $2.91 \mathrm{e}+02$ & $5.76 \mathrm{e}+03$ & $1.42 \mathrm{e}+01$ \\
Extended $f 10$ & $0.00 \mathrm{e}+00$ & $4.03 \mathrm{e}-07$ & $8.79 \mathrm{e}-03$ & $4.27 \mathrm{e}+02$ \\
Bohachevsky & $0.00 \mathrm{e}+00$ & $0.00 \mathrm{e}+00$ & $4.19 \mathrm{e}-02$ & $0.00 \mathrm{e}+00$ \\
Schaffer & $0.00 \mathrm{e}+00$ & $5.15 \mathrm{e}-07$ & $5.07 \mathrm{e}-03$ & $4.28 \mathrm{e}+02$ \\
$f 12$ & $0.00 \mathrm{e}+00$ & $3.09 \mathrm{e}-11$ & $3.61 \mathrm{e}+00$ & $8.42 \mathrm{e}+01$ \\
$f 13$ & $9.03 \mathrm{e}+00$ & $1.66 \mathrm{e}+01$ & $1.49 \mathrm{e}+02$ & $2.53 \mathrm{e}+02$ \\
$f 14$ & $0.00 \mathrm{e}+00$ & $9.24 \mathrm{e}-01$ & $4.75 \mathrm{e}+00$ & $3.89 \mathrm{e}+02$ \\
$f 15$ & $0.00 \mathrm{e}+00$ & $0.00 \mathrm{e}+00$ & $0.00 \mathrm{e}+00$ & $0.00 \mathrm{e}+00$ \\
$f 16$ & $0.00 \mathrm{e}+00$ & $9.23 \mathrm{e}-10$ & $3.70 \mathrm{e}+00$ & $1.97 \mathrm{e}+02$ \\
$f 17$ & $5.03 \mathrm{e}+00$ & $1.35 \mathrm{e}+01$ & $2.23 \mathrm{e}+01$ & $6.07 \mathrm{e}+02$ \\
$f 18$ & $0.00 \mathrm{e}+00$ & $2.78 \mathrm{e}-01$ & $2.37 \mathrm{e}+00$ & $2.34 \mathrm{e}+02$ \\
$f 19$ & $0.00 \mathrm{e}+00$ & $0.00 \mathrm{e}+00$ & $4.19 \mathrm{e}-02$ & $0.00 \mathrm{e}+00$ \\
Solved funcs. & 14 & 7 & 2 & 4 \\
\hline & & & & \\
\hline
\end{tabular}

Table 6 Average error on 500-D functions

\begin{tabular}{lllll}
\hline Function & MOS & MDE-DC & DE & MTS-LS1 \\
\hline Sphere & $0.00 \mathrm{e}+00$ & $0.00 \mathrm{e}+00$ & $2.46 \mathrm{e}+01$ & $5.77 \mathrm{e}-12$ \\
Schwefel 2.21 & $5.51 \mathrm{e}-04$ & $3.80 \mathrm{e}-04$ & $1.44 \mathrm{e}+02$ & $5.34 \mathrm{e}-06$ \\
Rosenbrock & $4.57 \mathrm{e}+01$ & $2.57 \mathrm{e}+01$ & $1.12 \mathrm{e}+05$ & $2.20 \mathrm{e}+02$ \\
Rastrigin & $0.00 \mathrm{e}+00$ & $1.02 \mathrm{e}-12$ & $1.63 \mathrm{e}+01$ & $5.62 \mathrm{e}-12$ \\
Griewank & $0.00 \mathrm{e}+00$ & $0.00 \mathrm{e}+00$ & $4.73 \mathrm{e}-01$ & $4.24 \mathrm{e}-03$ \\
Ackley & $0.00 \mathrm{e}+00$ & $0.00 \mathrm{e}+00$ & $1.06 \mathrm{e}+00$ & $6.18 \mathrm{e}-12$ \\
Schwefel 2.22 & $0.00 \mathrm{e}+00$ & $0.00 \mathrm{e}+00$ & $0.00 \mathrm{e}+00$ & $1.46 \mathrm{e}-12$ \\
Schwefel 1.2 & $1.28 \mathrm{e}+04$ & $1.09 \mathrm{e}+04$ & $6.70 \mathrm{e}+04$ & $6.16 \mathrm{e}+03$ \\
Extended $f 10$ & $0.00 \mathrm{e}+00$ & $3.41 \mathrm{e}+00$ & $1.12 \mathrm{e}-02$ & $1.00 \mathrm{e}+03$ \\
Bohachevsky & $0.00 \mathrm{e}+00$ & $0.00 \mathrm{e}+00$ & $2.93 \mathrm{e}-01$ & $0.00 \mathrm{e}+00$ \\
Schaffer & $0.00 \mathrm{e}+00$ & $3.36 \mathrm{e}+00$ & $2.43 \mathrm{e}-01$ & $1.00 \mathrm{e}+03$ \\
$f 12$ & $0.00 \mathrm{e}+00$ & $4.08 \mathrm{e}-01$ & $1.16 \mathrm{e}+01$ & $2.47 \mathrm{e}+02$ \\
$f 13$ & $3.78 \mathrm{e}+01$ & $4.84 \mathrm{e}+01$ & $4.02 \mathrm{e}+02$ & $5.05 \mathrm{e}+02$ \\
$f 14$ & $0.00 \mathrm{e}+00$ & $1.89 \mathrm{e}+00$ & $1.16 \mathrm{e}+01$ & $1.10 \mathrm{e}+03$ \\
$f 15$ & $0.00 \mathrm{e}+00$ & $1.39 \mathrm{e}-11$ & $4.19 \mathrm{e}-02$ & $1.08 \mathrm{e}-12$ \\
$f 16$ & $0.00 \mathrm{e}+00$ & $9.97 \mathrm{e}-01$ & $1.32 \mathrm{e}+01$ & $4.99 \mathrm{e}+02$ \\
$f 17$ & $1.21 \mathrm{e}+01$ & $2.76 \mathrm{e}+01$ & $6.94 \mathrm{e}+01$ & $7.98 \mathrm{e}+02$ \\
$f 18$ & $0.00 \mathrm{e}+00$ & $1.05 \mathrm{e}+00$ & $3.87 \mathrm{e}+00$ & $5.95 \mathrm{e}+02$ \\
$f 19$ & $0.00 \mathrm{e}+00$ & $0.00 \mathrm{e}+00$ & $8.39 \mathrm{e}-02$ & $0.00 \mathrm{e}+00$ \\
Solved funcs. & 14 & 7 & 1 & 2 \\
\hline & & & & \\
\hline
\end{tabular}

In order to provide a proper statistical validation of the results, Holm's, Hochberg's and Wilcoxon procedures have been used. In the case of the Wilcoxon test, the
Table 7 Average error on 1,000-D functions

\begin{tabular}{lllll}
\hline Function & MOS & MDE-DC & DE & MTS-LS1 \\
\hline Sphere & $0.00 \mathrm{e}+00$ & $1.02 \mathrm{e}-12$ & $3.71 \mathrm{e}+01$ & $1.15 \mathrm{e}-11$ \\
Schwefel 2.21 & $4.25 \mathrm{e}-01$ & $1.02 \mathrm{e}-01$ & $1.63 \mathrm{e}+02$ & $2.25 \mathrm{e}-02$ \\
Rosenbrock & $6.15 \mathrm{e}+01$ & $2.45 \mathrm{e}+01$ & $1.59 \mathrm{e}+05$ & $2.10 \mathrm{e}+02$ \\
Rastrigin & $0.00 \mathrm{e}+00$ & $2.95 \mathrm{e}+00$ & $3.47 \mathrm{e}+01$ & $1.15 \mathrm{e}-11$ \\
Griewank & $0.00 \mathrm{e}+00$ & $0.00 \mathrm{e}+00$ & $7.36 \mathrm{e}-01$ & $3.55 \mathrm{e}-03$ \\
Ackley & $0.00 \mathrm{e}+00$ & $3.01 \mathrm{e}-12$ & $8.70 \mathrm{e}-01$ & $1.24 \mathrm{e}-11$ \\
Schwefel 2.22 & $0.00 \mathrm{e}+00$ & $0.00 \mathrm{e}+00$ & $0.00 \mathrm{e}+00$ & $0.00 \mathrm{e}+00$ \\
Schwefel 1.2 & $1.94 \mathrm{e}+05$ & $1.45 \mathrm{e}+05$ & $3.15 \mathrm{e}+05$ & $1.23 \mathrm{e}+05$ \\
Extended $f 10$ & $0.00 \mathrm{e}+00$ & $1.32 \mathrm{e}+02$ & $6.26 \mathrm{e}-02$ & $1.99 \mathrm{e}+03$ \\
Bohachevsky & $0.00 \mathrm{e}+00$ & $0.00 \mathrm{e}+00$ & $1.67 \mathrm{e}-01$ & $0.00 \mathrm{e}+00$ \\
Schaffer & $0.00 \mathrm{e}+00$ & $1.34 \mathrm{e}+02$ & $4.42 \mathrm{e}-02$ & $1.99 \mathrm{e}+03$ \\
$f 12$ & $0.00 \mathrm{e}+00$ & $3.24 \mathrm{e}+01$ & $2.58 \mathrm{e}+01$ & $5.02 \mathrm{e}+02$ \\
$f 13$ & $8.80 \mathrm{e}+01$ & $2.41 \mathrm{e}+02$ & $8.24 \mathrm{e}+04$ & $8.87 \mathrm{e}+02$ \\
$f 14$ & $0.00 \mathrm{e}+00$ & $1.96 \mathrm{e}+01$ & $2.39 \mathrm{e}+01$ & $2.23 \mathrm{e}+03$ \\
$f 15$ & $0.00 \mathrm{e}+00$ & $0.00 \mathrm{e}+00$ & $2.11 \mathrm{e}-01$ & $0.00 \mathrm{e}+00$ \\
$f 16$ & $0.00 \mathrm{e}+00$ & $6.70 \mathrm{e}+01$ & $1.83 \mathrm{e}+01$ & $1.00 \mathrm{e}+03$ \\
$f 17$ & $2.25 \mathrm{e}+01$ & $2.19 \mathrm{e}+02$ & $1.76 \mathrm{e}+05$ & $1.56 \mathrm{e}+03$ \\
$f 18$ & $0.00 \mathrm{e}+00$ & $3.26 \mathrm{e}+01$ & $7.55 \mathrm{e}+00$ & $1.21 \mathrm{e}+03$ \\
$f 19$ & $0.00 \mathrm{e}+00$ & $0.00 \mathrm{e}+00$ & $2.51 \mathrm{e}-01$ & $0.00 \mathrm{e}+00$ \\
Solved funcs. & 14 & 5 & 1 & 4 \\
\hline & & & & \\
\hline & & & & \\
\hline 14 & & & & \\
& & & &
\end{tabular}

adjusted $p$ value is computed as described in García et al. (2009), to allow the comparison of multiple algorithms avoiding the Family-Wise Error (FWER) that multiple pair-wise comparisons could have introduced. The results of these tests are reported in Table 11, and show, for all of them, that there is statistical evidence to state that the MOS algorithm is better than any of the algorithms considered in the comparison.

Tables 9 and 10 present the average ranking of the comparison of MOS with the reference algorithms up to 500 and 1,000 dimensions, respectively. As it was remarked before, two different comparisons have been needed due to the lack of results for the G-CMA-ES algorithm in 1,000 dimensions (Table 11).

In both tables we can see that the best average ranking is obtained by the MOS-based algorithm, followed by the two DE algorithms, the G-CMA-ES algorithm and the worst results are for the $\mathrm{CHC}$ algorithm. The differences are especially important with the DE with binomial crossover, the G-CMA-ES and the CHC algorithms.

With these results in mind, the same statistical validation described in the previous paragraphs has been employed. Tables 12 and 13 present the results of this statistical validation up to 500 and 1,000 dimensions, respectively, which concludes that the algorithm presented in this work is statistically better than any of the reference algorithms considering any of the validation procedures. 
Table 8 Average ranking for the first comparison up to 1,000 dimensions

\begin{tabular}{ll}
\hline & Ranking \\
\hline MOS & 1.56 \\
MDE-DC & 2.09 \\
MTS-LS1 & 3.05 \\
DE & 3.31 \\
\hline
\end{tabular}

Table 9 Average ranking for the second comparison up to 500 dimensions

\begin{tabular}{ll}
\hline & Ranking \\
\hline MOS & 1.36 \\
DEExp & 2.18 \\
DEBin & 3.43 \\
G-CMA-ES & 3.70 \\
CHC & 4.32 \\
\hline
\end{tabular}

Table 10 Average ranking for the second comparison up to 1,000 dimensions

\begin{tabular}{ll}
\hline & Ranking \\
\hline MOS & 1.22 \\
DEExp & 1.94 \\
DEBin & 3.18 \\
CHC & 3.66 \\
\hline
\end{tabular}

\subsection{Scalability analysis}

In this section we analyze the scalability behavior of the proposed algorithm. In general, the MOS algorithm exhibits an excellent scalability behavior. In Tables 3, 4, $5,6,7$ it can be seen that our algorithm solves the same number of functions, 14, regardless their dimensionality (except in 100 dimensions, in which $f 14$ does not converge in 1 out of the 25 independent executions, as it was mentioned before). On the other hand, the other algorithms under study see how their performance degrades as the number of dimensions increases. For the five remaining functions, which are not solved at any dimension, the behavior of the proposed algorithm is different. The order of magnitude of the average error of the first group of functions, made up of Rosenbrock, $f 13$ and $f 17$ functions, grows linearly, although it is almost constant, with the number of dimensions (see Fig. 1). Both hybrid functions, $f 13$ ad $f 17$, have in common that they are combinations of Rosenbrock's function with another function, so it is normal that their scalability behavior is linked to that exhibited by the individual function. In this function, there is a very narrow valley that goes from local optimum to global optimum. Finding that valley is difficult and, for that reason, there is a high deviation in the average errors of different executions. However, reaching average regions of the solutions space should be relatively easy for a good search algorithm, which explains why the average errors remain more or less constant (or at least in similar orders of magnitude) regardless of the number of dimensions.

Regarding the other two functions, the order of magnitude of the average error for Schwefel's 1.2 seems to grow more or less logarithmically with the number of dimensions, whereas for Schwefel's problem 2.21 it is not clear at all. Both functions are unimodal and not separable nor easily optimized dimension by dimension. However, Bohachevsky's function shares the same characteristics (and Griewank's, except for the unimodality) and they can be solved by the MOS algorithm, so the difficulty issue should be at a different place.

If we pay more attention to Schwefel's problem 2.21, we can see that it presents large neutral areas, as the final fitness of solution is only determined by the value of one single dimension (the one with the highest absolute value). The effect of this characteristic is more pronounced as the number of dimensions is increased. Furthermore, the dimension with more influence in the final fitness value can change from one solution to another or when a solution is modified, so this information can not be exploited by the search algorithm, which makes more difficult to find an optimal solution. Both characteristics could explain the scalability behavior of the proposed algorithm on this function.

Considering now Schwefel's problem 1.2, we can see that, in this case, the influence of some components (the first ones, in particular) on the final fitness value is even more pronounced in this function than in the previous one. This probably explains why the average error quickly increases as the dimensionality of the problem grows, especially for the first numbers of dimensions that have been tested. When the number of dimensions increases up to $500-1,000$, the average errors are so large that the influence of this characteristic is not as important as in smaller dimensions.

\subsection{Running time analysis}

In the previous section we conducted a scalability analysis from the point of view of the average error. In this section, a similar analysis is carried out on the computational running time. For each function and dimension, the running time of the algorithm at each execution has been recorded and averaged. Tables 14 and 15 show this information, which is expressed in seconds. 
Table 11 Statistical validation for the first comparison (MOS is the control algorithm)

\begin{tabular}{|c|c|c|c|c|c|}
\hline MOS vs. & $z$ value & $p$ value & Holm $p$ value & Hochberg $p$ value & Wilcox $p$ value \\
\hline MDE-DC & $2.84 \mathrm{e}+00$ & $4.54 \mathrm{e}-03$ & $4.54 \mathrm{e}-03^{*}$ & $4.54 \mathrm{e}-03^{*}$ & $5.55 \mathrm{e}-04^{*}$ \\
\hline MTS-LS1 & $7.95 \mathrm{e}+00$ & $1.78 \mathrm{e}-15$ & $3.55 \mathrm{e}-15^{*}$ & $3.55 \mathrm{e}-15^{*}$ & $8.42 \mathrm{e}-10^{*}$ \\
\hline $\mathrm{DE}$ & $9.33 \mathrm{e}+00$ & $0.00 \mathrm{e}+00$ & $0.00 \mathrm{e}+00^{*}$ & $0.00 \mathrm{e}+00^{*}$ & $6.80 \mathrm{e}-15^{*}$ \\
\hline \multicolumn{5}{|c|}{ Wilcox $p$ value with FWER: MOS vs. MDE-DC, MTS-LS1, DE } & $5.55 \mathrm{e}-04^{*}$ \\
\hline
\end{tabular}

${ }^{*}$ Means that there are statistical differences with significance level $\alpha=0.05$

Table 12 Statistical validation for the second comparison up to 500 dimensions (MOS is the control algorithm)

\begin{tabular}{|c|c|c|c|c|c|}
\hline MOS vs. & $z$ value & $p$ value & Holm $p$ value & Hochberg $p$ value & Wilcox $p$ value \\
\hline $\mathrm{CHC}$ & $1.15 \mathrm{e}+01$ & $0.00 \mathrm{e}+00$ & $0.00 \mathrm{e}-00^{*}$ & $0.00 \mathrm{e}-00^{*}$ & $1.80 \mathrm{e}-14^{*}$ \\
\hline DEExp & $3.21 \mathrm{e}+00$ & $1.35 \mathrm{e}-03$ & $1.35 \mathrm{e}-03^{*}$ & $1.35 \mathrm{e}-03^{*}$ & $5.96 \mathrm{e}-09^{*}$ \\
\hline DEBin & $8.05 e+00$ & $8.88 \mathrm{e}-16$ & $1.78 \mathrm{e}-15^{*}$ & $1.78 \mathrm{e}-15^{*}$ & $3.54 \mathrm{e}-13^{*}$ \\
\hline G-CMA-ES & $9.13 e+00$ & $0.00 \mathrm{e}+00$ & $0.00 \mathrm{e}+00^{*}$ & $0.00 \mathrm{e}+00^{*}$ & $7.764 \mathrm{e}-11^{*}$ \\
\hline \multicolumn{5}{|c|}{ Wilcox $p$ value with FWER: MOS vs. CHC, DEExp, DEBin, G-CMA-ES } & $6.04 \mathrm{e}-09^{*}$ \\
\hline
\end{tabular}

* Means that there are statistical differences with significance level $\alpha=0.05$

Table 13 Statistical validation for the second comparison up to 1,000 dimensions (MOS is the control algorithm)

\begin{tabular}{llllll}
\hline MOS vs. & $z$ value & $p$ value & Holm $p$ value & Hochberg $p$ value & Wilcox $p$ value \\
\hline CHC & $1.30 \mathrm{e}+01$ & $0.00 \mathrm{e}+00$ & $0.00 \mathrm{e}+00^{*}$ & $0.00 \mathrm{e}+00^{*}$ & $1.30 \mathrm{e}-1.7^{*}$ \\
DEExp & $3.85 \mathrm{e}+00$ & $1.18 \mathrm{E}-04$ & $1.18 \mathrm{e}-04^{*}$ & $1.18 \mathrm{e}-04^{*}$ & $3.87 \mathrm{e}-11^{*}$ \\
DEBin & $1.05 \mathrm{e}+01$ & $0.00 \mathrm{e}+00$ & $0.00 \mathrm{e}+00^{*}$ & $0.00 \mathrm{e}+00^{*}$ & $2.35 \mathrm{e}-16^{*}$ \\
Wilcox $p$ value with FWER: MOS vs. CHC, DEExp, DEBin & & & $3.87 \mathrm{e}-11^{*}$ \\
\hline
\end{tabular}

* Means that there are statistical differences with significance level $\alpha=0.05$

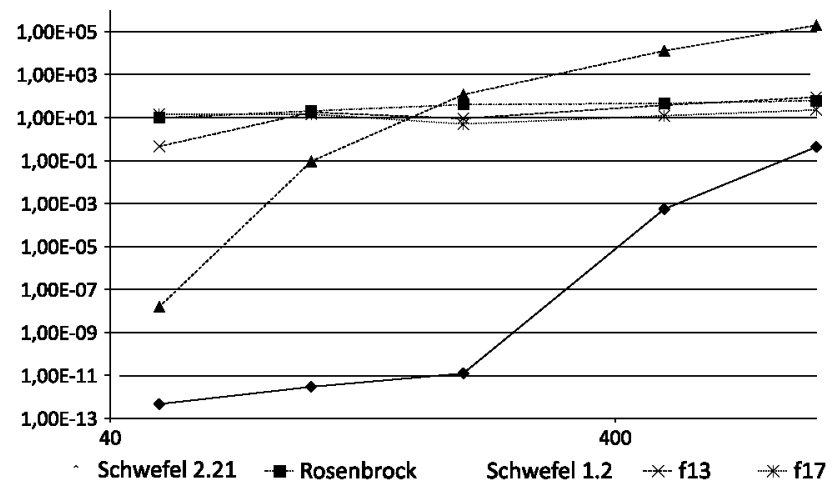

Fig. 1 Scalability plots for MOS in logarithmic scale

Figure 2 plots the information gathered in Tables 14 and 15. In this figure, the computational running time as the number of dimensions grows has been represented for all the functions. It can be seen that the required computational time seems to grow quadratically with the number of dimensions of the function. Using the Big Theta notation, we could say that the computational time of the MOS algorithm $(f(n))$ is $\Theta\left(n^{2}\right)$, being $k_{1}$ and $k_{2}$ two constants such as the following expression is satisfied: $k_{1} \cdot n^{2} \leq f(n) \leq k_{1} \cdot n^{2}$

The values for these two constants can be roughly be established to $\frac{1}{1400}$ and $\frac{1}{400}$, respectively. These values are relatively large, and they help to soften the effect of the quadratic function. In conclusion, the running time of the proposed algorithm seems to scale well up to a large number of dimensions.

\subsection{Participation and quality analysis}

We conclude the analysis of the proposed algorithm by studying its dynamic behavior with regard to the adjustment of the participation of each technique on the overall search process and the selection of the effective quality function to be used at each moment.

Regarding the adjustment of the participation of the two considered techniques, we have observed three different behaviors:

- Clear dominance of the DE technique. This happens with Ackley, $f 12, f 14, f 16, f 18$ and Sphere.

- Clear dominance of the MTS-LS1 technique. This is the case for Schwefel 1.2. 
Table 14 Running time (in seconds) of the MOS algorithm for 50, 100 , and $200 \mathrm{D}$

\begin{tabular}{llll}
\hline Function & $50 \mathrm{D}$ & $100 \mathrm{D}$ & $200 \mathrm{D}$ \\
\hline Sphere & $6.74 \mathrm{e}-01$ & $1.87 \mathrm{e}+00$ & $6.58 \mathrm{e}+00$ \\
Schwefel 2.21 & $6.16 \mathrm{e}-01$ & $1.40 \mathrm{e}+00$ & $3.43 \mathrm{e}+00$ \\
Rosenbrock & $5.06 \mathrm{e}-01$ & $1.64 \mathrm{e}+00$ & $6.87 \mathrm{e}+00$ \\
Rastrigin & $8.06 \mathrm{e}-01$ & $2.73 \mathrm{e}+00$ & $1.06 \mathrm{e}+01$ \\
Griewank & $1.06 \mathrm{e}+00$ & $3.16 \mathrm{e}+00$ & $1.28 \mathrm{e}+01$ \\
Ackley & $9.79 \mathrm{e}-01$ & $3.15 \mathrm{e}+00$ & $1.14 \mathrm{e}+01$ \\
Schwefel 2.22 & $7.41 \mathrm{e}-01$ & $2.12 \mathrm{e}+00$ & $7.01 \mathrm{e}+00$ \\
Schwefel 1.2 & $2.16 \mathrm{e}-01$ & $7.37 \mathrm{e}-01$ & $2.91 \mathrm{e}+00$ \\
Extended $f 10$ & $5.45 \mathrm{e}+00$ & $2.13 \mathrm{e}+01$ & $8.50 \mathrm{e}+01$ \\
Bohachevsky & $1.18 \mathrm{e}+00$ & $3.84 \mathrm{e}+00$ & $1.66 \mathrm{e}+01$ \\
Schaffer & $5.45 \mathrm{e}+00$ & $2.08 \mathrm{e}+01$ & $9.89 \mathrm{e}+01$ \\
$f 12$ & $1.82 \mathrm{e}+00$ & $7.13 \mathrm{e}+00$ & $2.87 \mathrm{e}+01$ \\
$f 13$ & $1.80 \mathrm{e}+00$ & $8.25 \mathrm{e}+00$ & $2.78 \mathrm{e}+01$ \\
$f 14$ & $2.01 \mathrm{e}+00$ & $8.24 \mathrm{e}+00$ & $3.29 \mathrm{e}+01$ \\
$f 15$ & $8.30 \mathrm{e}-01$ & $2.77 \mathrm{e}+00$ & $9.89 \mathrm{e}+00$ \\
$f 16$ & $3.10 \mathrm{e}+00$ & $1.17 \mathrm{e}+01$ & $4.95 \mathrm{e}+01$ \\
$f 17$ & $4.34 \mathrm{e}+00$ & $1.74 \mathrm{e}+01$ & $6.95 \mathrm{e}+01$ \\
$f 18$ & $4.40 \mathrm{e}+00$ & $1.71 \mathrm{e}+01$ & $7.72 \mathrm{e}+01$ \\
$f 19$ & $1.06 \mathrm{e}+00$ & $3.83 \mathrm{e}+00$ & $1.45 \mathrm{e}+01$ \\
\hline
\end{tabular}

Table 15 Running time (in seconds) of the MOS algorithm for 500D and $1,000 \mathrm{D}$

\begin{tabular}{lll}
\hline Function & $500 \mathrm{D}$ & $1,000 \mathrm{D}$ \\
\hline Sphere & $3.85 \mathrm{e}+01$ & $1.42 \mathrm{e}+02$ \\
Schwefel 2.21 & $1.84 \mathrm{e}+01$ & $6.57 \mathrm{e}+01$ \\
Rosenbrock & $4.23 \mathrm{e}+01$ & $1.47 \mathrm{e}+02$ \\
Rastrigin & $7.22 \mathrm{e}+01$ & $2.46 \mathrm{e}+02$ \\
Griewank & $1.19 \mathrm{e}+02$ & $4.32 \mathrm{e}+02$ \\
Ackley & $6.68 \mathrm{e}+01$ & $2.44 \mathrm{e}+02$ \\
Schwefel 2.22 & $4.08 \mathrm{e}+01$ & $1.43 \mathrm{e}+02$ \\
Schwefel 1.2 & $1.50 \mathrm{e}+01$ & $5.80 \mathrm{e}+01$ \\
Extended $f 10$ & $5.36 \mathrm{e}+02$ & $2.07 \mathrm{e}+03$ \\
Bohachevsky & $9.08 \mathrm{e}+01$ & $3.11 \mathrm{e}+02$ \\
Schaffer & $6.27 \mathrm{e}+02$ & $2.04 \mathrm{e}+03$ \\
$f 12$ & $1.62 \mathrm{e}+02$ & $6.43 \mathrm{e}+02$ \\
$f 13$ & $1.86 \mathrm{e}+02$ & $6.58 \mathrm{e}+02$ \\
$f 14$ & $1.77 \mathrm{e}+02$ & $7.16 \mathrm{e}+02$ \\
$f 15$ & $5.46 \mathrm{e}+01$ & $1.93 \mathrm{e}+02$ \\
$f 16$ & $2.90 \mathrm{e}+02$ & $1.11 \mathrm{e}+03$ \\
$f 17$ & $4.43 \mathrm{e}+02$ & $1.61 \mathrm{e}+03$ \\
$f 18$ & $4.55 \mathrm{e}+02$ & $1.64 \mathrm{e}+03$ \\
$f 19$ & $8.11 \mathrm{e}+01$ & $3.01 \mathrm{e}+02$ \\
\hline & &
\end{tabular}

- Collaboration of both techniques (to a greater or a lesser extent, sometimes with the dominance of one of them but with a representative participation of the other

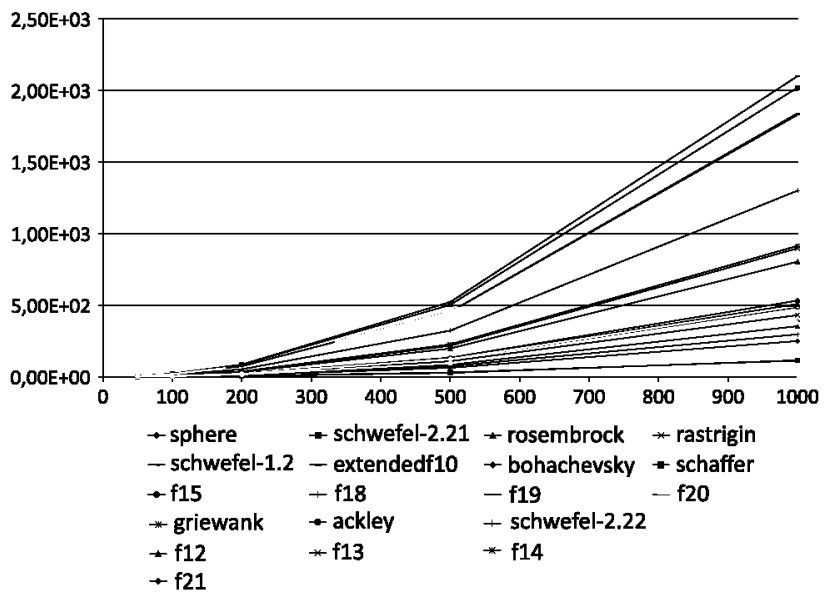

Fig. 2 Computational running time (in seconds) for all the functions

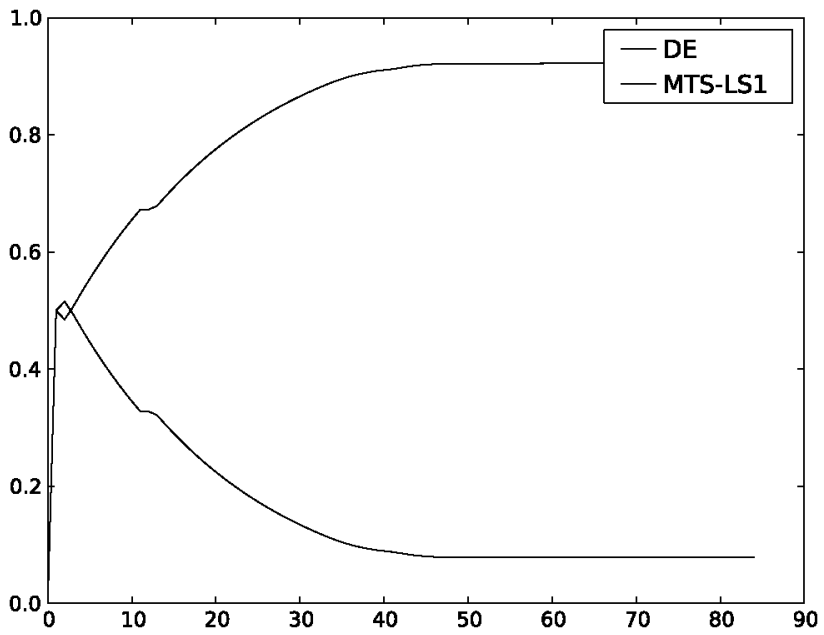

Fig. 3 Participation adjustment for Ackley in 50 dimensions

one through all the search). This happens with Bohachevsky, Extended $f 10, f 13, f 15, f 17, f 19$, Griewank, Rastrigin, Rosenbrock, Schaffer, Schwefel 2.21 and Schwefel 2.22.

An example of this behavior is depicted in Figs. 3, 4 and 5 , respectively.

On the other hand, regarding the selection of the active quality function, we can conduct a similar analysis. In this case, the following three groups have been identified:

- Functions for which the average fitness increment quality function is mostly used: Ackley, Bohachevsky, Extended $f 10, f 12, f 14, f 15, f 16, f 18, f 19$, Griewank, Rastrigin, Schaffer, Schwefel 2.22 and Sphere.

- Functions for which the raw number of fitness increments is mostly used: Schwefel 1.2 and Schwefel 2.21.

- Functions for which both quality functions alternate: $f 13, f 17$ and Rosenbrock. 


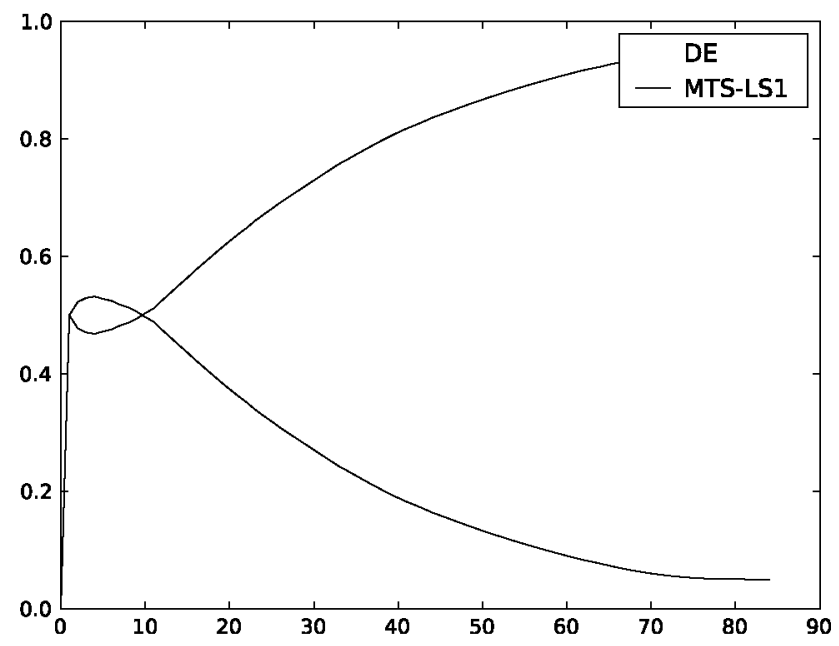

Fig. 4 Participation adjustment for Schwefel 1.2 in 50 dimensions

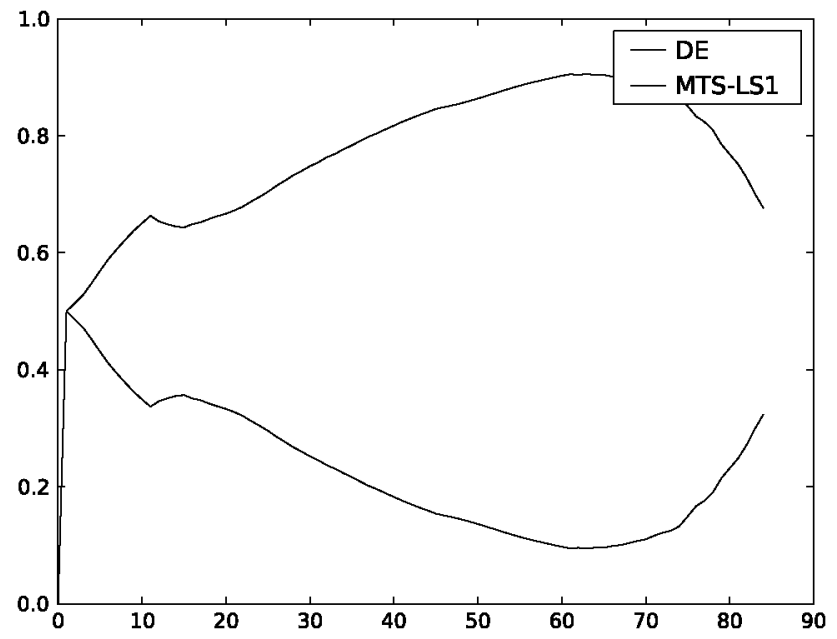

Fig. 5 Participation adjustment for $f 17$ in 50 dimensions

It can be seen that, for most of the functions, the average fitness increment is preferred to guide the adjustment of the participation of the techniques. However, there are some specific functions for which it is important to conduct small changes on the solutions rather than large modifications with important fitness increments.

The active quality function for one function of each group is depicted in Figs. 6, 7 and 8, respectively.

\section{Conclusions}

In this work, a new hybrid memetic algorithm based on the MOS framework has been presented and thoroughly tested on a large set of scalable continuous functions. Different numbers of dimensions have been tested to study the scalability behavior of the algorithm. The hybrid algorithm

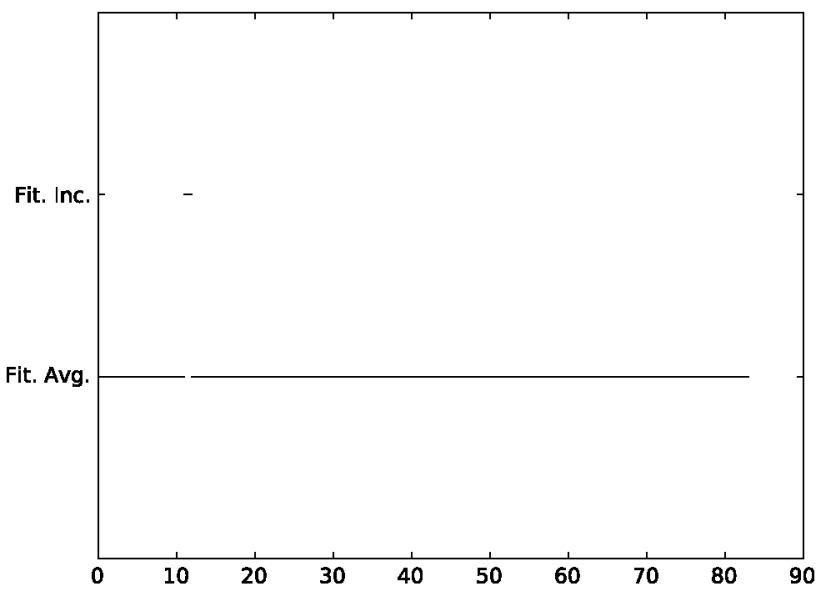

Fig. 6 Active quality function for Ackley in 1,000 dimensions

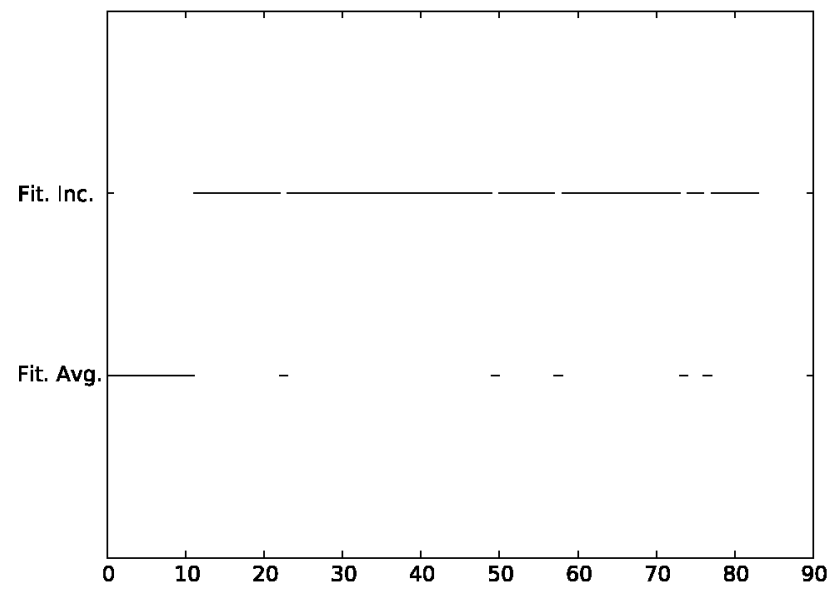

Fig. 7 Active quality function for Schwefel 1.2 in 1,000 dimensions

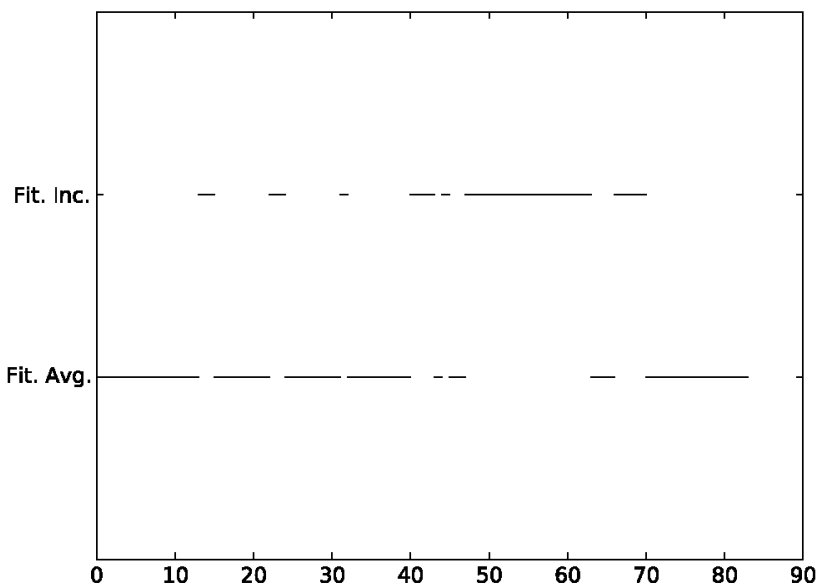

Fig. 8 Active quality function for Rosenbrock in 1,000 dimensions

has been statistically compared with each of its composing algorithms, as well as with a static combination of both algorithms. All the considered statistical tests found 
significant differences, which means that the MOS-based algorithm outperforms all the other algorithms. The same validation procedure has been conducted to compare our approach with several reference algorithms, classic in the literature of continuous optimization (CHC, two DEs and G-CMA-ES). Once again, the statistical tests found significant differences between the MOS-based algorithm and all the other algorithms. This allows us to state that the algorithm presented in this work is better than any of these reference approaches for this benchmark.

Regarding the scalability issue, the proposed algorithm has been able to keep a stable behavior regardless the dimensionality of the problem. 14 out of the 19 functions of the benchmark have been solved to the maximum possible precision in all the considered dimensions. For the remaining functions, the order of magnitude of the average error grows differently, with only one function with a relatively bad scalability behavior.

Finally, the computational running time has also been examined. The results show that the required computational time grows more or less quadratically. However, this time is scaled by a factor that reduces the actual computational time to reasonable values up to relatively large numbers of dimensions.

Acknowledgments This work was supported by the Madrid Regional Education Ministry and the European Social Fund, financed by the Spanish Ministry of Science TIN2007-67148 and supported by the Cajal Blue Brain Project. The authors thankfully acknowledge the computer resources, technical expertise and assistance provided by the Centro de Supercomputación y Visualización de Madrid (CeSViMa) and the Spanish Supercomputing Network.

\section{References}

Caponio A, Cascella G, Neri F, Salvatore N, Sumner M (2007) A fast adaptive memetic algorithm for on-line and off-line control design of PMSM drives. IEEE Trans Syst Man Cybern Part B 37:28-41

Caponio A, Neri F, Cascella G, Salvatore N (2008) Application of memetic differential evolution frameworks to PMSM drive design. In: Proceedings of the 2008 IEEE congress on evolutionary computation, CEC 2008 (IEEE World Congress on Computational Intelligence), pp 2113-2120

Caponio A, Neri F, Tirronen V (2009) Super-fit control adaptation in memetic differential evolution frameworks. Soft Comput Fusion Found Methodol Appl 13(8):811-831

Caruana R, Schaffer J (1988) Representation and hidden bias: Gray vs. binary coding for genetic algorithms. In: Proceedings of the 5th international conference on machine learning, ICML 1998, pp 153-161

Gao Y, Wang Y-J (2007) A memetic differential evolutionary algorithm for high dimensional functions' optimization. In: Proceedings of the third international conference on natural computation (ICNC 2007), pp 188-192

García S, Molina D, Lozano M, Herrera F (2009) A study on the use of non-parametric tests for analyzing the evolutionary algorithms behaviour: a case study on the CEC2005 special session on real parameter optimization. J Heurisics 15(6):617-644

Grefenstette J (1986) Optimization of control parameters for genetic algorithms. IEEE Trans Syst Man Cybern 16(1):122-128

LaTorre A (2009) A framework for hybrid dynamic evolutionary algorithms: multiple offspring sampling (mos). Ph.D. thesis, Universidad Politécnica de Madrid (November 2009)

LaTorre A, Peña J, Muelas S, Freitas A (2010) Learning hybridization strategies in evolutionary algorithms. Intell Data Anal 14(3)

Lin G, Kang L, Chen Y, McKay B, Sarker R (2007) A self-adaptive mutations with multi-parent crossover evolutionary algorithm for solving function optimization problems. In: Kang L, Zeng YLS (eds) Advances in computation and intelligence: proceedings of the 2nd international symposium, ISICA 2007. Lectures notes in computer science, vol 4683/2007, pp 157-168

Mladenovic N, Hansen P (1997) Variable neighborhood search. Comput Oper Res 24(11):1097-1100

Muelas S, LaTorre A, Peña J (2009) A memetic differential evolution algorithm for continuous optimization. In: Proceedings of the 9th international conference on intelligent systems design and applications, ISDA 2009, pp 1080-1084

Ong Y-S, Keane A (2004) Meta-lamarckian learning in memetic algorithms. IEEE Trans Evol Computat 8(2):99-110

Qin A, Suganthan P (2005) Self-adaptive differential evolution algorithm for numerical optimization. In: Proceedings of the IEEE congress on evolutionary computation, CEC 2005, pp 1785-1791

Rönkkönen J, Kukkonen S, Price K (2005) Real-parameter optimization with differential evolution. In: Proceedings of the IEEE congress on evolutionary computation, CEC 2005, pp 506-513

Schnier T, Yao X (2000) Using multiple representations in evolutionary algorithms. In: Proceedings of the 2 nd IEEE congress on evolutionary computation, CEC 2000, vol 1, pp 479-486

Suganthan P, Hansen N, Liang J, Deb K, Chen Y, Auger A, Tiwari S (2005) Problem definitions and evaluation criteria for the cec 2005 special session on real-parameter optimization. Tech. Rep. 2005005, 1School of EEE. Nanyang Technological University and Kanpur Genetic Algorithms Laboratory (KanGAL)

Talbi E-G (2002) A taxonomy of hybrid metaheuristics. J Heuristics $8(5): 541-564$

Tang K, Yao X, Suganthan P, MacNish C, Chen Y, Chen C, Yang Z (2007) Benchmark functions for the cec 2008 special session and competition on large scale global optimization. Tech. rep., Nature Inspired Computation and Applications Laboratory, USTC

Thierens D (2005) An adaptive pursuit strategy for allocating operator probabilities. In: Proceedings of the 7th genetic and evolutionary computation conference, GECCO 2005, pp 1539-1546

Tirronen V, Neri F, Karkkainen T, Majava K, Rossi T (2007) A memetic differential evolution in filter design for defect detection in paper production. In: Proceedings of EvoWorkshops 2007, pp 330-339

Tirronen V, Neri F, Kärkkäinen T, Majava K, Rossi T (2008) An enhanced memetic differential evolution in filter design for defect detection in paper production. Evol Comput 16(4):529-555

Tseng L, Chen C (2008) Multiple trajectory search for large scale global optimization. In: Proceedings of the 10th IEEE congress on evolutionary computation, CEC 2008 (IEEE World Congress on Computational Intelligence). IEEE Press, pp 3052-3059

Whitacre J, Pham T, Sarker R (2006) Credit assignment in adaptive evolutionary algorithms. In: Proceedings of the 8th genetic and evolutionary computation conference, GECCO 2006, Seattle, pp 1353-1360

Wolpert D, Macready W (1997) No free lunch theorems for optimization. IEEE Trans Evol Comput 1(1):67-82 